\title{
Las relaciones cambiantes de un patido de ámbito no estatal (PANE) con su partido hermano: el caso de Iniciativa per Catalunya Verds (1987-2008)
}

\section{Tània Verge Mestre}

Universitat Pompeu Fabra. Departament de Ciències Polítiques i Socials tania.verge@upf.edu

\section{Resumen}

Para analizar las relaciones entre los partidos de ámbito estatal (PAE) y los partidos de ámbito no estatal (PANE) que actúan como partidos hermanos, resulta útil emplear las dimensiones que miden el grado de integración vertical entre los niveles de un partido en sistemas multinivel. El caso de estudio es el marco de cooperación entre ICV e IU vigente en el período 1987-2007, el más antiguo y con la autonomía más elevada de los tres que existen en España. El análisis de las relaciones interpartidistas permite comprender también el desarrollo ideológico y organizativo de ICV en las últimas décadas.

Palabras clave: partidos políticos, partidos de ámbito no estatal, gobierno multinivel, ICV, IU.

Abstract. The changing relationships between a non-state wide party and its sister party: the case of Iniciativa per Catalunya Verds (1987-2007)

In order to analyse the relationships between state-wide parties (SWP) and non-state wide parties (NSWP) which act as sister parties it is useful to apply the dimensions that measure the degree of vertical integration between the state and sub-state levels of party organization in multilevel contexts. The case studied is that of the relationships between ICV and IU in the period 1987-2007, the oldest and loosest interparty cooperation formula of the three that exist in Spain. The analysis of the interparty relationships allows us to understand too the ideological and organizational evolution of ICV in the last decades.

Key words: political parties, non state-wide parties, multilevel government, ICV, IU.

\section{Sumario}

Introducción: gobierno multinivel y partidos políticos

Primera fase: una década de tensiones

$(1987-1997)$

La ruptura de relaciones (1997-2002)
El reencuentro (2003-2008)

Conclusiones

Referencias bibliográficas

Listado de acrónimos 


\section{Introducción: gobierno multinivel y partidos políticos}

La adaptación organizativa de los partidos que compiten en contextos de gobierno multinivel se ha erigido en una línea de investigación en auge en la última década. La descentralización política empuja a los partidos a regionalizar no sólo sus estrategias y programas, sino también su propia estructura (Pallarés y Keating, 2003: 243; Chhibber y Kollman, 2004; Roller y Van Houten, 2003; Hopkin y Bradbury, 2006). Las relaciones entre el nivel estatal y el subestatal de la organización constituyen, entonces, una compleja arena de incentivos e interacciones en la lucha por recursos, liderazgos y autonomía en la toma de decisiones (Deschower, 2003; Thorlakson, 2006).

Existe un abanico de fórmulas de relación muy amplio entre las unidades estatal y subestatales de un partido en torno al grado de integración vertical, definida como los vínculos organizativos y las fórmulas de cooperación entre dichos niveles (Thorlakson, 2001: 2; Janda, 1980; Smiley, 1987; Astudillo y GarcíaGuereta, 2005; Méndez y Orte, 2005; Ramiro-Fernández y Pérez-Nievas, 2005). El estudio de la integración vertical puede acometerse a partir del análisis de tres dimensiones principales:

1. Las cuestiones organizativas (centralización en la toma de decisiones, financiación o representación del nivel subestatal en los órganos centrales del partido).

2. La formulación de la oferta programática.

3. El diseño de las estrategias electorales y la política de alianzas.

La determinación del grado de integración vertical entre ambos niveles permite ubicar a los partidos en un continuum de mayor a menor centralización de las decisiones en las distintas dimensiones presentadas (Thorlakson, 2001: 6-7). Cuando la integración es total entre la unidad estatal y la subestatal, se trata de partidos unitarios. La categoría diametralmente opuesta la encontramos en los partidos truncados, cuando no existe ninguna contraparte en el otro nivel de gobierno. Entre estos dos extremos, caben varias posibilidades. En los partidos integrados y los confederales, el grado de integración vertical es elevado (la diferencia radica en el nivel donde resida la autonomía más amplia). Finalmente, en los partidos truncados, existen pocos vínculos y escasa cooperación entre la unidad estatal y la subestatal ${ }^{1}$.

El incentivo para la descentralización organizativa resulta mayor en países con partidos subestatales nacionalistas y, dentro de estos países, en los territorios donde la competición política se halla más regionalizada por la existencia de fuertes identidades duales que tienen su expresión en formaciones políticas que buscan representar los intereses de la región. En el caso español, podría esperarse, pues, que, en aquellas comunidades autónomas con una identificación nacional más intensa, como Cataluña, Galicia, el País Vasco y, 
por extensión, Navarra, las federaciones de los partidos de ámbito estatal (PAE) disfrutaran de una mayor autonomía, es decir, que la integración vertical entre los dos niveles del partido fuera más laxa. Sin embargo, en algunas de estas regiones o bien no se observa un grado de autonomía mayor de la unidad subestatal o bien el PAE (PSOE, PP o IU) ha establecido relaciones de cooperación con partidos de ámbito no estatal (PANE): el PP, con la Unión del Pueblo Navarro (UPN); el PSOE, con el PSC, e IU, con Iniciativa per Catalunya Verds $(\mathrm{ICV})^{2}$.

La atención prestada a aquellos PANE que mantienen relaciones estables con un PAE y que actúan en la región en la que compiten electoralmente como partidos hermanos ha sido más bien escasa (Roller y Van Houten, 2003; Thorburn, 1991; Renzsch, 2001). En este artículo, planteamos que, para analizar las relaciones entre los PAE y los PANE que mantienen una relación estable más o menos formalizada, resulta útil emplear las dimensiones que miden el grado de integración vertical entre los niveles de un mismo partido. Con esta metodología, nos planteamos descubrir qué tipo de integración vertical establecieron IU e ICV y cómo evolucionó ésta a lo largo del tiempo. Simultáneamente, la ilustración de este caso de estudio nos permite plantear algunas hipótesis sobre las variables que condicionan una mayor o menor integración vertical, que deberán ser comprobadas con el análisis de otros casos.

En este artículo, aplicaremos el esquema de análisis propuesto en el caso de ICV en el período comprendido entre 1987, momento fundacional del partido, y 2008. El interés del caso radica en que se trata del marco de cooperación interpartidista más antiguo y con la autonomía más elevada de los tres que pueden encontrarse en España. Además, las relaciones entre ICV e IU y los partidos que las crearon, el PSUC y el PCE, respectivamente, han sido cambiantes desde su propio establecimiento.

Por otro lado, el análisis de estas relaciones nos permite examinar y comprender el desarrollo ideológico y organizativo de ICV desde su creación. La evolución de ICV estuvo marcada en los años ochenta y noventa por la estrategia política de IU. La falta de acuerdo en las dimensiones arriba mencionadas separó progresivamente a ambos partidos, hasta consumarse la ruptura de las relaciones históricas que habían mantenido el PCE y el PSUC. Con el distanciamiento de su partido hermano, ICV emprendió una nueva senda ideológica y organizativa que le permitió convertirse en un partido exitoso a nivel electoral y gubernamental. Paradójicamente, en la siguiente década, los mismos motivos que produjeron el rechazo de las estrategias electorales y de la evolución programática de ICV son los que llevaron a la actual dirección de IU a plantear un nuevo acercamiento a su antiguo socio y a emular algunas de sus estrategias.

2. Por razones de simplicidad, a lo largo del artículo utilizaremos exclusivamente las siglas ICV para referirnos a este partido, aún siendo necesario reconocer la evolución en su denominación: IC, de 1987 a 1998; IC-V, de 1998 a 2002, e Iniciativa per Catalunya Verds (ICV) desde entonces. 
El artículo se organiza de la siguiente forma. La primera sección analiza cada una de las dimensiones presentadas en la primera década de relaciones entre ambos partidos. La segunda sección da cuenta de los motivos que condujeron a la ruptura entre ICV e IU y de las estrategias desarrolladas por ICV en esos momentos. En la siguiente sección, se examina el restablecimiento de relaciones entre las dos formaciones y se analiza cómo ICV se ha convertido en un modelo de referencia para IU, al que ha intentado emular en diferentes aspectos, en especial, en los programáticos, electorales y de alianzas. La última sección presenta las principales conclusiones.

\section{Primera fase: una década de tensiones (1987-1997)}

Joan Saura, presidente de ICV desde 2000, afirmava tras ser elegido: «La soberanía de ICV, nuestra política de unidad de las izquierdas y nuestra apuesta por un proyecto ecosocialista y de radicalidad democrática estuvieron en el origen de la ruptura con Izquierda Unida; pero esto que fueron elementos de crisis hoy son elementos de futuro para ICV» (Saura, 2000: 7). Con estas palabras, el líder de ICV ponía de manifiesto que las relaciones entre ambas formaciones, estructuradas bajo dimensiones tales como la autonomía organizativa («la soberanía de ICV»), la política de alianzas («nuestra política de unidad de las izquierdas») y la formulación de la oferta programática («nuestra apuesta por un proyecto ecosocialista y de radicalidad democrática») se tensaron de tal modo que alcanzaron un punto de no retorno: la ruptura total de relaciones y el desgajamiento de una parte de ICV afín a los postulados de la dirección de IU, lo que implicaría la competición electoral entre los dos partidos.

Veamos, a continuación, cual ha sido el grado de autonomía en cada una de las dimensiones seleccionadas en el período 1987-1997 (cuestiones organizativas, definición de la oferta programática y estrategias electorales y política de alianzas), etapa en la que existía un marco de cooperación entre ambas formaciones.

\section{La autonomía organizativa}

Para examinar el grado de autonomía organizativa entre los dos partidos, cabe analizar, en primer lugar, el tipo de vínculos históricos mantenidos entre ambos, ya que el origen de los partidos puede determinar su evolución posterior (Panebianco, 1988). Las tensiones por la autonomía entre IU e ICV recogían y ampliaban las existentes entre el PCE y el PSUC prácticamente desde la creación del último, en 1936, como la fusión de los partidos comunistas y socialistas catalanes. Debido a la histórica fusión, el PCE no tenía en Cataluña una federación regional, sino un partido hermano que, además, también de forma excepcional y exclusiva, fue admitido como miembro de pleno derecho de la Tercera Internacional (Estruch, 1982: 52).

Las condiciones del exilio y la clandestinidad provocaron inicialmente la sucursalización del PSUC. La integración vertical se basaba en el principio de 
reciprocidad. Varios miembros de la dirección del PSUC participaban con plenos derechos en el Comité Central del PCE y dos miembros de la dirección del PCE eran miembros, a su vez, de la del PSUC. Por lo tanto, los acuerdos tomados por el primero eran vinculantes para el segundo. Aunque, en 1956, el PSUC cambió el estatus del PCE en los órganos y congresos propios al de invitado, en la práctica, ambas direcciones se hallaban íntimamente ligadas, por lo que este hecho no resultó problemático durante años (Cebrián, 1997: 54). La tensión se agravó con la participación en la competición democrática a partir de 1977, en la que el PSUC obtuvo unos excelentes resultados. La dirección del PSUC se negó entonces a la propuesta del PCE de añadir entre paréntesis las siglas de su partido a las del PSUC, como hacían los socialistas (Vega y Erroteta, 1982: 76). Ambos partidos formaron un grupo parlamentario de tipo federal, es decir, los comunistas catalanes no quedaban meramente integrados en el grupo estatal. Además, el PSUC empezó a rechazar cualquier cláusula que le obligara a cumplir los acuerdos del PCE no coincidentes con los acuerdos propios, rechazo que tuvo su reflejo en los estatutos del partido en 1981 . La integración vertical existente en esos momentos era moderadamente baja, de manera que podía ubicarse el marco de cooperación establecido en el continuum presentado en una posición próxima a los partidos confederales.

Las elecciones de 1982, en las que el PCE pasó de 15 a 3 diputados, provocaron la dimisión de Santiago Carrillo, que fue sustituido por Gerardo Iglesias. El secretario general del PSUC, Antoni Gutiérrez Díaz, resistió hasta 1986, cuando los resultados en las elecciones generales fueron pésimos, y tomó el relevo Rafael Ribó, para quien, entre los principales errores del PSUC, se encontraban la renuncia a hacer una política propia respecto al PCE, que nunca reflexionó sobre «lo que significa ser un partido comunista en una realidad plurinacional» ${ }^{3}$. Su perfil más catalanista marcó el distanciamiento definitivo. Las relaciones mejoraron levemente tras la dimisión de Carrillo, pero empeoraron de nuevo bajo la dirección de Julio Anguita en el PCE (desde 1988) y en IU (desde 1989).

En 1986, el PCE lideró la constitución de una nueva plataforma que aglutinaba a diferentes pequeños partidos de izquierda (IU) y el PSUC siguió sus pasos en Cataluña con la creación de $\mathrm{ICV}^{4}$. El cambio en la coyuntura política en la que ambos partidos relanzaban sus proyectos para superar la crisis electoral que sufrían facilitó la introducción de nuevas reglas. En este contexto, la dirección del PSUC y de ICV buscaron definir ex novo una relación de igual a igual con IU (Rius, 2005: 43).

Los vínculos entre IU e ICV desde sus inicios tuvieron un carácter más débil que los que mantenían el PCE y el PSUC, los principales partidos de las nuevas formaciones. A ICV sólo se le otorgó la representación con voz en los órganos de IU y, en cualquier caso, la asistencia de miembros de ICV en estos

3. Entrevista de Manuel Vázquez Montalbán a Rafael Ribó (1988: 94-97).

4. Para un análisis de los motivos que condujeron al lanzamiento de IU e ICV y sus procesos de creación, véase Botella (2003), Rius (2005) y Ramiro-Fernández (2004). 
órganos fue más bien irregular. Por su parte, de manera informal, ICV reconocía la presencia de miembros de IU en sus órganos, aunque ésta fue también prácticamente nula ${ }^{5}$. Nunca se implantaron mecanismos de coordinación entre las dos organizaciones, pese a que, de acuerdo con el «principio de federalidad» estatutario ${ }^{6}$, IU debía diseñar un marco de relaciones estables con ICV para coordinar la definición de políticas y estrategias bajo un mismo proyecto federal a nivel estatal. A su vez, la única referencia a IU en las normas de funcionamiento de ICV era implícita cuando éste último hablaba de forma general de las relaciones con otros partidos de ámbito estatal ${ }^{7}$. ICV dejaba también claro que, como fuerza política soberana, cualquier forma de cooperación estable con otra debía circunscribirse a la fórmula de confederación ${ }^{8}$. Además, los diputados electos se integraban en un grupo parlamentario federal que respetaba la soberanía de sus integrantes y en el que se distribuían la subvención pública entre los dos partidos.

A principios de 1991, se había consensuado un protocolo de relaciones que, aunque fue aprobado en los respectivos comités ejecutivos, nunca se llegó a firmar por desacuerdo de la dirección de IU sobre un modelo que consagraba la autonomía total de ICV (Rius, 2005: 44). En 1993, Ribó pasó a ser portavoz adjunto en el grupo parlamentario para buscar una mayor coordinación en la acción política entre ambos partidos. A partir de 1994, las profundas discrepancias que a menudo trascendían a la prensa, obligaron a ambos partidos a reflexionar sobre sus relaciones. La IV Asamblea Federal de IU acordó definir unas nuevas bases ${ }^{9}$, para lo cual se creó la Comisión IU-ICV, a fin de que trabajara en esta dirección. La IV Asamblea Nacional de ICV (1996) recogía también algunas reflexiones al respecto ${ }^{10}$, pero el agravamiento de las hostilidades desatado poco después impidió consolidar esta cooperación. Precisamente la falta de puentes comunicativos más allá del grupo parlamentario precipitó un final tan dramático, ya que las mayorías en ambas organizaciones empezaron a ver a los grupos minoritarios como una influencia externa del otro partido, que buscaba interferir en los procesos internos (Botella, 2003: 62).

\section{La formulación de la oferta programática}

ICV declaró, en sus primeras normas de funcionamiento, la soberanía para la elaboración de sus principios ideológicos y programáticos ${ }^{11}$. En cambio, IU e ICV convenían encuentros programáticos para la elaboración de las propues-

5 Informe político, IV Asamblea Nacional de ICV (1996), p. 29.

6. Estatutos III Asamblea Federal de IU (1990).

7. Manifest d'IC, p. 5, aprobado en la I Asamblea Nacional de ICV (1990).

8. Protocol de funcionament, apartado 9 (1990).

9. Disposición transitoria primera, Estatutos IV Asamblea Federal de IU (1994).

10. Informe político IV Asamblea Nacional de ICV (1996), p. 31.

11. Protocol de funcionament, preámbulo 1.1 (1990). 
tas políticas para el conjunto del Estado ${ }^{12}$. Así, ICV podía influir en la política de IU, pero no a la inversa.

Las diferencias en el contenido de la oferta programática defendida por cada partido están estrechamente relacionadas con su distinta naturaleza interna: coalición en el caso de IU y federación en el caso de ICV. El primero se constituyó en 1986 como una coalición de partidos que pactaron un sistema de cuotas en órganos directivos, listas electorales y reparto de recursos internos con una clara preeminencia del PCE, que mantenía activas todas sus funciones (excepto la electoral). En cambio, en ICV la distribución de recursos y cargos se acometía en base al consenso y no a partir de cuotas partidistas. Aunque el líder del PSUC se convirtió, como en el caso de IU, en el líder del nuevo partido, su dirección decidió "congelarlo» y canalizar toda su actividad a través de ICV. El traspaso formal de competencias (elaboración programática, ámbito institucional, proyección pública y relaciones con la sociedad) se produjo en el marco de la I Asamblea Nacional de ICV, celebrada en $1990^{13}$. Así, mientras que el PCE mantenía su carácter como partido independiente dentro de $\mathrm{IU}^{14}$, el PSUC devino gradualmente una estructura adormecida dentro de ICV.

La hegemonía del PCE en IU, reforzada por el progresivo abandono del resto de partidos fundadores de la coalición, condicionó así la evolución ideológica del nuevo partido. En cuanto a ICV, a las tradicionales demandas de la izquierda, se sumaban reivindicaciones nacionales de autogobierno. Por otro lado, la presencia de grupos pacifistas, feministas y ecologistas en su seno y el creciente apoyo obtenido entre el electorado de clase medias, profesionales liberales, funcionarios, intelectuales y jóvenes, motivaron el desarrollo de un nuevo proyecto político que integrara a las distintas sensibilidades (Botella, 2003: 62). En la búsqueda de una nueva identidad, Rafael Ribó se alineó con el Partido Comunista Italiano, que transitó hacia una organización postcomunista. Aunque estaba latente desde finales de 1991, fue a partir de 1996 cuando ICV pasó a definirse como ecosocialista ${ }^{15}$ (Gomà y Rius, 2006: 254).

En la política estatal, ICV mantuvo una política más pragmática en cuestiones como el Tratado de Maastricht. En octubre de 1992, los tres diputados catalanes votaron a favor del mismo y rechazaron la celebración de un referéndum. A diferencia de IU, los diputados de ICV votaron a favor del acuer-

12. Informe político, II Asamblea Federal de IU, p. 22; Normas de Funcionamiento de ICV, 1996, artículo 107.

13. Formalizado posteriormente en la XI Conferencia Nacional del PSUC ( 20 de diciembre de 1990).

14. Desde la creación de IU, la dirección del PCE discutió en ocasiones la posibilidad de disolver el partido. En 1991, cuando una sección de la dirección lo propuso, Anguita amagó con dimitir. Se temía que la disolución del PCE generara una fuerte división en el partido. Por otro lado, se consideraba que el avance electoral de IU no hacía necesaria la disolución del PCE (Ramiro-Fernández, 2005).

15. Véanse los Principios básicos para un programa de izquierdas ecosocialista en la transición al siglo XXI (V Asamblea Nacional de ICV, 1998). 
do sobre pensiones y la reforma laboral negociados por el Gobierno y los sindicatos mayoritarios (Dunphy, 2004). De este modo, desde ICV se respetaba la independencia sindical en su estrategia de negociación con el Gobierno, mientras que IU consideraba que los sindicatos debían adoptar una oposición frontal al Gobierno socialista desde una perspectiva ideologizada (Rius, 2005: 15). Un resultado de estos desacuerdos fue el retraso de aproximadamente un año y medio en la transferencia de la proporción de los fondos públicos que correspondía a los diputados de ICV.

\section{La politica de alianzas}

La política de alianzas de IU e ICV no se basaba solamente en la autonomía, sino también en una evidente y temprana diferenciación entre ambas. Como describe Botella (2003: 62), durante los años ochenta, ICV tuvo como objetivo contribuir a desarrollar distintos partidos de izquierda en varias comunidades autónomas para, posteriormente, federarlas. Así, por ejemplo, en 1988, en lugar de apoyar a la federación vasca de IU, ICV participó en la campaña de Euzkadiko Eskerra, lo cual molestó evidentemente a la dirección de su partido hermano, aunque no llegó a estallar ninguna crisis.

Otro objetivo de la política de alianzas de ICV fue el establecimiento de una coordinación estable con las federaciones de IU en la Comunidad Valenciana (EUPV) y en las Islas Baleares (EUIB), organizaciones con las que buscaba acordar un marco estable de relaciones y de coordinación de las políticas comunes, a partir del reconocimiento de la existencia de vínculos históricos y culturales con otras comunidades de los Países Catalanes ${ }^{16}$. No deja de ser un hecho más bien insólito que un partido establezca relaciones con sólo algunas de las federaciones de su partido hermano.

El siguiente proyecto en el que se embarcó ICV fue en el establecimiento de alianzas electorales con partidos verdes. La ausencia de un partido verde relevante en el mercado electoral español dejaba margen para intentar conquistar un espacio que se mostraba rendible en otros países europeos. Se trataba, además, de un proyecto fallido por parte de IU, que nunca había conseguido una coalición estable con los verdes a nivel estatal ni autonómico ${ }^{17}$. En las elecciones locales y autonómicas de 1995 y en las generales de 1996, concurrió en coalición con pequeños partidos verdes, con lo cual consiguió un éxito relativo que empujó a sus dirigentes a proseguir en esa dirección.

Sin embargo, la política de alianzas que desató una mayor confrontación y que coadyuvó determinantemente en la ruptura de relaciones fue la relación con el PSOE. La estrategia de IU en los años ochenta y noventa se basaba en el rechazo a toda colaboración con el PSOE, a quien identificaba con la dere-

16. Informe político, IV Asamblea Nacional de ICV (1996), p. 32. Ya mencionado en la I Asamblea Nacional.

17. De hecho, en las elecciones generales y europeas celebradas en 2004, Los Verdes concurrieron en las listas del PSOE. 
cha (la teoría de «las dos orillas»), incluso tras las elecciones de 1996, con lo cual se anulaba cualquier posibilidad de alcanzar pactos. Además, para IU, los pactos entrañaban el riesgo de quedar reducida a una fuerza subordinada del PSOE que, a largo plazo, condujera a su desaparición. Se creía que esta dura oposición permitiría al partido convertirse en la formación hegemónica en la izquierda e incluso adelantar electoralmente a su máximo rival, la estrategia del Sorpasso (Ramiro-Fernández, 2004: 15). Esta posición era contestada en el seno de la coalición por una corriente de opinión, Nueva Izquierda (NI), que apoyaba la aproximación con los socialistas.

La posición de ICV se aproximaba más a la de NI que a la de la dirección de IU y calificaba la tesis de las dos orillas de «maniquea y excluyente» (Ribó, 1999: 140). Desde la soberanía en política de alianzas declarada ya desde la I Asamblea Nacional (1990), Ribó planteó, a mediados de los años noventa, un proceso de convergencia entre el «centro-izquierda, la izquierda transformadora y el nacionalismo progresista» ${ }^{18}$, refiriéndose a un encuentro entre el PSC, ICV y ERC basada en la teoría del «olivo», alianzas entre partidos del ámbito de la izquierda ya acometidas en varios países europeos como Italia, Suecia, Finlandia, Francia, Irlanda y la todavía en preparación coalición roji-verde en Alemania (Ribó, 1999: 84). Ribó planteaba, así, recuperar el sentido de l'Entesa, candidatura senatorial conjunta presentada en 1977 (integrada por el PSUC, el PSC, ERC, Estat Català e independientes) que se impuso por mayoría absoluta en todas las circunscripciones. Los socialistas no debían ser los principales rivales políticos, sino los gobiernos conservadores de los distintos niveles de gobierno (el PP en el gobierno central y CiU en la Generalitat). ICV mantenía y ampliaba sus pactos con el PSC en el ámbito local y en 1997 incluso sondeó al PSC para fijar las prioridades de un futuro gobierno autonómico de izquierdas y dejó abierta la posibilidad de alcanzar un pacto preelectoral, así como de presentar listas conjuntas en las elecciones locales de 1999.

El marco de relaciones interpartidistas entre el PAE y el PANE entre 1987 y 1997 muestra una elevada autonomía para el segundo. Existen pocos vínculos y escasa cooperación entre la unidad estatal y la subestatal, y las discrepancias frecuentes e intensas en las cuestiones programáticas, así como en la política de alianzas, denotan un bajo nivel de integración vertical. En este caso, podríamos afirmar que este marco de cooperación se asemeja al de un partido bifurcado.

\section{La ruptura de relaciones (1997-2002)}

En 1997, la federación gallega de IU, afín a la corriente Nueva Izquierda, alcanzó un pacto electoral con los socialistas y los verdes. La dirección de IU montó en cólera y, ante el fracaso en la consecución del apoyo del 30\% de la militancia para convocar una asamblea extraordinaria que eligiera a una nueva

18. Informe político, IV Asamblea Nacional de ICV (1996), p. 12. 
dirección, decidió crear una nueva federación del partido en este territorio. Asimismo, purgó las direcciones de otras federaciones, como la valenciana, que habían prestado su apoyo a los líderes de Galicia y disolvió las direcciones de las federaciones de Cantabria y Castilla-La Mancha (Paniagua y RamiroFernández, 2003). ICV confrontó radicalmente esta decisión y ayudó a la federación gallega en la campaña electoral. El desacuerdo no sólo era estratégico (sobre la conveniencia de alcanzar pactos con el PSOE), sino también debido a la inexcusable interferencia en la autonomía de la federación sancionada.

IU reaccionó rompiendo relaciones con ICV y expulsando a los parlamentarios catalanes al grupo mixto. Más de cincuenta años de relación entre el PSUC y el PCE se habían dinamitado. Hubo, sin embargo, un sector del partido que discrepaba con la mayoría de ICV y que ya se había mostrado crítico con la «hibernación» del PSUC, la colaboración con los socialistas y el deterioro de las relaciones con IU. Se trataba del Colectivo Rojo, Verde y Violeta, una corriente de opinión creada en 1993 (inicialmente denominada Plataforma de Izquierdas). Esta corriente, junto al Partit dels Comunistes de Catalunya $(\text { PCC })^{19}$ y a una parte escindida del PSUC (el PSUC viu ${ }^{20}$, encontraron el apoyo de Anguita para impulsar la creación de una nueva plataforma que actuase como el nuevo referente de IU en Cataluña. Esquerra Unida i Alternativa (EUiA) nacía finalmente en mayo de 1998.

En esta arriesgada decisión, la de fracturar el partido hermano en dos listas, pudo ser relevante el hecho que, en las dos últimas elecciones generales (1993 y 1996) y autonómicas (1995), los resultados medios de IU en el conjunto del Estado fueron sistemáticamente mejores que los de ICV, lo cual hacía ver a la dirección de IU que era ella la que defendía las políticas y las estrategias que mejor satisfacían a su electorado (véase la tabla 1). Así, el coste esperado de la ruptura podía preverse como un mal menor. En este sentido, la voluntad de IU de controlar a ICV se explicaría, a partir de Downs (1998), por la combinación de una baja volatilidad y moderados niveles de localización (similitud entre los resultados electorales obtenidos por ambos partidos en elecciones generales y autonómicas). La unidad estatal tuvo, entonces, fuertes incentivos para centralizar las decisiones. Como ICV no se avino a las políticas y estrategias de IU, el desacuerdo conllevó la ruptura definitiva, con lo cual pasó a ser un partido truncado.

Las dos formaciones pasaron a presentarse por separado en las siguientes convocatorias electorales y obtuvieron resultados desastrosos, aunque dispares. Las expectativas de EUiA no se confirmaron. A pesar de representar el $32 \%$ de los delegados de ICV justo antes de la ruptura, su líder, Antoni Lucchetti, creía que dos tercios del electorado roji-verde apoyarían a la nueva

19. El PCC, miembro fundador de IC, abandonó la federación en 1989 por desacuerdo con el proceso de unidad comunista emprendido en esas fechas, pero, desde 1993, colaboraba con IC y concurría en coalición con la misma a las elecciones.

20. El PSUC viu reconstruyó las relaciones que el PSUC había mantenido con el PCE hasta la década de los ochenta. 
Tabla 1. Porcentaje de votos y diputados en elecciones generales y autonómicas ${ }^{1}$

\begin{tabular}{lcclcc}
\hline Año & $\begin{array}{c}\text { Media IU } \\
\text { autonómicas }\end{array}$ & IU & ICV & $\begin{array}{c}\text { Diferencia } \\
\text { en votos }^{1}\end{array}$ & $\begin{array}{c}\text { Proporción } \\
\text { sobre escaños }\end{array}$ \\
\hline 1986 G & - & $4,6(7)$ & $3,9(1)$ & $-0,7$ & 14,3 \\
1988 A & $5,8(51)$ & - & $7,8(9)$ & 2,0 & 17,6 \\
1989 G & - & $9,1(14)$ & $7,3(3)$ & $-1,7$ & 17,6 \\
\hline 1991 A & $6,3(60)$ & - & $6,6(7)$ & 0,3 & 11,7 \\
1993 G & - & $9,5(15)$ & $7,5(3)$ & $-2,1$ & 16,7 \\
1995 A & $12,0(66)$ & - & $9,8(1)$ & $-2,2$ & 16,7 \\
1996 G & - & $10,5(19)$ & $7,6(2)$ & $-2,9$ & 9,5 \\
1999 A & $5,9(28)$ & - & $3,92(5)$ & $-3,4$ & 17,8 \\
2000 G & - & $5,4(8)$ & $3,5(1)$ & $-1,9^{2}$ & -3 \\
\hline 2003 A & $5,7(34)$ & - & $7,34(9)$ & 1,6 & 26,5 \\
2004 G & - & $4,9(3)$ & $5,8(2)$ & 0,8 & 40,0 \\
2006-2007 A & $5,7(30)$ & - & $9,5(12)$ & 3,8 & 40,0 \\
2008 G & - & $3,8(1)$ & $4,9(1)$ & 0,8 & 50,0 \\
\hline
\end{tabular}

1. Los valores positivos indican una ventaja de ICV sobre IU.

2. Votos obtenidos en exclusiva por ICV.

3. ICV se incorporó al grupo parlamentario mixto.

4. Desde 2003, ICV y EUiA han concurrido juntos en todas las convocatorias electorales.

5. Además de IU e ICV-EUiA, también integra el grupo ERC.

coalición ${ }^{21}$. Pero en las elecciones autonómicas de 1999, mientras que ICV obtuvo 122.895 votos $(3,9 \%)$ y 5 escaños $^{22}$, los 44.454 votos de EUiA resultaron insuficientes para asegurarse representación institucional. Según una encuesta realizada por ICV, 100.000 de los 350.000 electores que apoyaron al partido en 1995 desertaron tanto de ICV como de EUiA tras su ruptura ${ }^{23}$.

La misma tendencia se manifestó en las elecciones generales de 2000. ICV vio reducida su representación a un diputado tras lograr 119.290 votos $(3,5 \%)$. EUiA, con 75.091 votos (2,2\%), no consiguió representación. En conjunto, las dos organizaciones recibieron el 5,8\% de los votos, ligeramente por encima de la media estatal de IU (5,4\%). También en 2000, ICV formó candidatura senatorial con el PSC y ERC, l'Entesa Catalana de Progrés, que repitió en 2004 y 2008.

Debido a la falta de una contraparte estatal, ICV promovió la creación, en 1999, de una coalición de pequeños PANE en Aragón, Galicia y Andalucía, para concurrir juntos a las elecciones europeas, que, por escaso margen no obtuvo un eurodiputado. No obstante, esta tarea legitimó al partido ante la Federación Europea de los Verdes, que lo admitió como miembro en 2002. En este período, también se consolidaron las relaciones con el Grupo Parlamentario Verde del Parlamento Europeo. Por otro lado, en 2001, ICV

21. Véase El País, 21 de agosto de 1997.

22. ICV concurrió en coalición con el PSC en Tarragona, Lleida y Girona.

23. Véase El Pais, 2 de julio de 2002. En las elecciones europeas de 1999, ICV formó coalición con Los Verdes, la Chunta Aragonesista, Esquerda Galega e Izquierda Andaluza. 
fue el principal impulsor de la creación de la federación de partidos Los VerdesIzquierda Verde, cuya coordinación asumió y contribuyó a la constitución de partidos verdes en el resto del Estado, con el objetivo de repetir candidatura en las siguientes elecciones europeas. Además de consolidar las relaciones con el ecologismo político, ICV pretendía, por un lado, mostrar a IU que tenía capacidad de organización a nivel estatal (Rius, 2005: 20) y, por otro, "superar la visión centralista del Estado compartida por PSOE e IU [...]. Se trata de construir un proyecto político confederal en el que cada organización va a conservar sus siglas y su soberanía» ${ }^{24}$. No obstante, los modestos resultados electorales disolvieron implícitamente la federación, que, además, en 2004, había permitido a sus miembros pactar coaliciones con distintos partidos en cada territorio. En 2004, ICV se convirtió en uno de los partidos fundadores del Partido Verde Europeo.

\section{El reencuentro (2003-2008)}

Tras los malos resultados electorales cosechados por IU en 2000, EUiA, con un manifiesto apoyo de IU, planteó un acercamiento a ICV para establecer una coalición de cara a las siguientes elecciones generales, locales y autonómicas. La aproximación se veía facilitada por el cambio de liderazgo, en el año 2000, en los tres partidos implicados: Joan Saura sustituyó a Rafael Ribó en ICV; Jordi Miralles, a Antoni Luchetti en EUiA, y Gaspar Llamazares, a Julio Anguita en $\mathrm{IU}^{25}$.

La coalición ICV-EUiA, acordada en 2002, experimentó una recuperación significativa en las elecciones autonómicas de 2003, lo cual elevó su representación a nueve diputados, gracias a los 241.183 votos obtenidos. En este contexto, por primera vez, los tres partidos de izquierdas del Parlament (PSC, ERC e ICV) alcanzaron un acuerdo para formar gobierno. La coalición ICVEUiA obtuvo las carteras de Medio Ambiente y Vivienda y de Relaciones Institucionales y Participación Ciudadana.

La participación en la coalición tripartita se ha valorado como un medio para reforzar la utilidad de ICV ante el electorado y para garantizar la elaboración de políticas de izquierda ${ }^{26}$. Por el contrario, la evaluación de esta primera experiencia gubernamental por parte de EUiA fue más crítica, puesto que consideraba que había predominado la desigualdad, tanto en la toma de decisiones como en el reparto de cargos ${ }^{27}$. De hecho, sólo uno de los trece parlamentarios (en las distintas instituciones) y dos representantes en el segundo nivel de

24. Jaume Bosch, responsable en esos momentos de Acción Política de IC-V, «La izquierda verde, un nuevo espacio», El Mundo, 20 de mayo de 2001.

25. Tras el mandato interino de Francisco Frutos durante unos meses, desde el ingreso hospitalario de Anguita hasta la celebración de la asamblea federal de IU en 2000.

26. Estrategia politica de la izquierda verde nacional, VIII Asamblea Nacional de ICV (2004), p. 29-30.

27. Informe político, IV Asamblea Nacional de EUiA (2005), p. 89. 
gobierno de la Generalitat correspondieron a EUiA. Algunas inequidades fueron corregidas en 2006, tras la reedición del tripartito, cuando dos candidatos de EUiA fueron ubicados en posiciones seguras en las listas, se le dio más visibilidad en la denominación de la coalición y obtuvo más cargos institucionales. La coalición ICV-EUiA incrementó su peso relativo al añadir a sus carteras la de Interior, así como la responsabilidad en las negociaciones de l'Estatut con el gobierno central.

Mientras que ICV vivía su momento más dulce, IU se hallaba sumida en una fuerte crisis electoral desde las elecciones locales y europeas de 1999 (véase la tabla 1). En las elecciones generales del 2000, IU redujo a la mitad su proporción de votos $(5,4 \%)$ y sólo obtuvo ocho diputados, pese al pacto preelectoral alcanzado con los socialistas ${ }^{28}$. IU retornaba al techo electoral que el PCE obtuvo en 1982. Esta derrota conllevó inevitablemente un cambio en la coalición dominante, como se ha adelantado. Gaspar Llamazares se impuso a Francisco Frutos, secretario general del PCE desde 1998, por un escaso margen, lo cual forzó al nuevo líder a integrar a miembros de la lista derrotada en la dirección ${ }^{29}$ (Verge, 2007). Se rompía así la tradicional asociación entre dirigentes del PCE y de IU, y ello produjo una situación asimétrica en la que la facción dominante del PCE estaba en minoría en la dirección de IU, mientras que la facción dominante de IU era minoritaria en la del PCE.

El núcleo duro del PCE mostraba una oposición frontal a las medidas propuestas por Llamazares para relanzar la formación. En primer lugar, Llamazares había intentado desradicalizar algunas propuestas tradicionales comunistas y transformar a IU en una organización ecosocialista, siguiendo el modelo de ICV. De acuerdo con la facción crítica del PCE, este cambio constituía una amenaza al corazón de la identidad del partido. El ecosocialismo se consideraba una traición al pasado comunista y una aproximación a valores cuasisocialdemócratas que no garantizan una recuperación electoral ${ }^{30}$. Las políticas moderadas de los partidos verdes privilegiarían ser un partido de gobierno a ser un partido de lucha y de movilización social ${ }^{31}$ (Verge, 2007).

En segundo lugar, las alianzas con otras fuerzas políticas, en particular con el PSOE, también han sido fuente de una dura confrontación. Para la coalición dominante de IU, la aceptación de una «relación preferencial» con el gobierno socialista entre 2004 y 2008, ha permitido al partido desarrollar una oposición «influyente y exigente», con el objetivo de forzar al Gobierno a ela-

28. Incluía una breve lista de políticas, el compromiso de formar un gobierno de coalición si el PSOE no obtenía mayoría absoluta y un pacto en las candidaturas senatoriales en 8 de las 54 provincias.

29. Llamazares se impuso de nuevo ante el sector crítico en las asambleas de 2003 y 2004 , y obtuvo la reelección.

30. Entrevista con el ex secretario de organización de IU y miembro del comité ejecutivo de IU y del PCE.

31. Entrevista con un ex miembro del comité ejecutivo de IU y del PCE. 
borar políticas de izquierda ${ }^{32}$. En cambio, el PCE considera que esta estrategia desdibuja la identidad de IU y la subsume en el espacio socialista, ya que el apoyo prestado no se ha acompañado de acuerdos programáticos ${ }^{33}$.

Este sector se ha mostrado también crítico con las experiencias de gobierno a nivel autonómico de las federaciones de IU en Asturias, Euskadi y también de la coalición ICV-EUiA en Cataluña, todo lo contrario de la posición mantenida por Llamazares para quien el avance de estas federaciones es visto como un buen resultado para IU en su conjunto, puesto que podría contribuir a su relanzamiento político y electoral ${ }^{34}$. Es decir, la tensión entre partido de lucha y partido de gobierno se resuelve con la esperanza de que la potencial deserción de electores ideológicamente más comprometidos se compense con un incremento en el apoyo de votantes flotantes que valoren de forma positiva la actuación responsable del partido.

De este modo, el actual coordinador general ha buscado potenciar el perfil de gobierno de la formación en el nivel regional, para presentarse ante el electorado como una fuerza política útil y responsable y ha respaldado a las directivas de las federaciones regionales con participación en gobiernos autonómicos y con alianzas con otros partidos de izquierdas o nacionalistas que se veían acosadas por la oposición frontal del PCE y sus ramas regionales, apoyando incluso una significativa erosión de la integración vertical del partido ${ }^{35}$ (Verge, 2007). La victoria de Llamazares en las elecciones primarias forzadas por la dirección del PCE (noviembre de 2007) para elegir al cabeza de cartel de las elecciones generales de 2008 reforzó la posición de este sector para «refundar el proyecto de IU», su apertura a nuevos sectores como el ecologista, el pacifista y el feminista y la colaboración con otras fuerzas de la izquierda alternativa o nacionalista, lo que sectores críticos interpretan como la voluntad de "cargarse definitivamente la IU roja e inventarse una IU ecosocialista» ${ }^{36}$.

Ante la debilidad electoral de IU, ICV se vio fortalecida. Cabe recordar que, en las elecciones generales de 2004, mientras que IU obtuvo un $5 \%$ de votos y 3 diputados (dos por Madrid y una por Valencia), la coalición ICVEUiA aglutinó el 5,8\% de los votos y consiguió dos diputados, los dos miembros de ICV. Representaba, pues, el 40\% del reeditado grupo parlamentario federal, una posición muy influyente. La colaboración de ICV con los socialistas catalanes sin duda contribuía a aumentar el pragmatismo en el grupo y a evi-

32. Informe político, VIII Asamblea Federal de IU (2004).

33. Informe al Comité Federal del PCE, Bases politicas y organizativas para la recuperación de Izquierda Unida (18 de junio de 2006).

34. Resolución de la Presidencia Ejecutiva Federal de IU (6 de noviembre de 2006).

35. En los últimos años, las federaciones de Asturias y de Euskadi han cambiado su estatus en relación con el conjunto del partido. Mientras que Ezker Batua Berdeak declaró su independencia de la formación (con lo cual adquirió un estatus similar al de EUiA), Izquierda Xunida de Asturias pasó de definirse como parte integrante de IU a una organización que, desde su plena soberanía, decide federarse con IU. Ambas decisiones contaron con el apoyo de Llamazares.

36. El Pais, 3 de diciembre de 2007. 
tar tensiones en la «relación preferencial» mantenida con el Gobierno. El grupo parlamentario cuenta con dos portavocías, una para IU (Gaspar Llamazares) y otra para ICV (Joan Herrera), y se comparte su dirección. También ha permitido a IU dar más énfasis a lo «verde». Un claro ejemplo es la propia denominación del grupo que estuvo vigente hasta marzo de 2006, Izquierda VerdeIU-ICV 37 , lo que conseguía superar la oposición interna en IU. La propuesta lanzada por Llamazares en la Asamblea Federal de diciembre de 2004 de incorporar «Izquierda Verde» a la denominación del partido encontró una oposición frontal y fue derrotada.

Por otro lado, el peso de ICV se hizo notar también en la elaboración de las candidaturas al Parlamento Europeo en 2004. ICV consiguió la segunda posición en la lista (con un apoyo de tan sólo el 68\% de la ejecutiva de IU) y su titular, una vez elegido, se integró en el Grupo de los Verdes, pese a que IU es parte del Grupo de la Izquierda Unida Europea-Izquierda Verde Nórdica.

En 2008, tanto IU como ICV sufrieron un nuevo retroceso electoral. IU perdió dos escaños e ICV, uno. Como resultado, cada partido cuenta con un diputado, lo cual equilibra las fuerzas en el grupo parlamentario compartido, al que se añadió ERC para facilitar su constitución.

Por último, la mejoría de relaciones con IU no ha sido óbice para que ICV se desvincule de la construcción del espacio de izquierda verde en el conjunto del Estado, aprovechando y mejorando la experiencia (fallida) de la federación de Los Verdes-Izquierda Verde. Actualmente, coopera con los Verdes del País Valencià y les Illes Balears y con las federaciones de IU de estos territorios (EUPV y EUIB, respectivamente), con las que se propone incrementar los encuentros institucionales y los acuerdos de cooperación. Además, ha creado el instituto Triangle de Mar Blava, en el marco de la fundación de estudios del partido, Nous Horitzons, destinado a promover la relación con las organizaciones políticas cercanas a ICV y las entidades sociales y culturales de las comunidades autónomas mencionadas. Por otro lado, para suplir la insuficiente articulación a nivel de encuadramiento político de la izquierda verde en numerosos puntos del territorio estatal, se plantea la creación, allí donde exista demanda, de agrupaciones de «amigos y amigas de ICV» (la denominación para los simpatizantes), que celebrarán reuniones de discusión política, recibirán información de ICV y podrán hacer llegar sus propuestas a los órganos de dirección ${ }^{38}$.

El ejemplo más paradigmático de este tipo de relaciones con algunas federaciones de IU es la establecida con EUPV. Además de caer en el ámbito de acción territorial de referencia para el partido, no es casual la implicación en esta región, ya que la federación valenciana de IU ha sido utilizada como cabeza

37. Al manifestar algunos líderes de ICV que fuera de Cataluña pedirían el voto para Los Verdes, tras una reunión con ICV, la Comisión Permanente de IU solicitó el cambio de nombre del grupo.

38. Una organització arrelada a la societat $i$ amb capacitat de govern, documento aprobado por la VIII Asamblea Nacional, p. 74. 
de martillo contra la dirección federal por parte del PCE. Esta relación se inició con la presencia pública de ICV en la campaña electoral de Compromís pel País Valencià, coalición de EUPV impulsada por la corriente de opinión Esquerra i País (que agrupaba en torno al 25\% de la organización), el Bloc Nacionalista Valencià y diversos grupos verdes valencianos. En octubre de 2007, Esquerra i País se convertía en partido político y el nombre escogido denotaba sus afinidades: Iniciativa del Poble Valencià (IPV). Además, IPV se declara como una fuerza ecosocialista, como izquierda verde valencianista. El vicepresidente de ICV, Jaume Bosch, manifestó todo su apoyo al proyecto valenciano homónimo y se mostró satisfecho ante el hecho de que su formación tenga un referente explícito en el País Valenciano. Esta simbiosis de intereses fue compartida inicialmente por el sector de Llamazares, es decir, se trataba de una estrategia de alcance federal ${ }^{39}$.

\section{Conclusiones}

En este artículo, se han examinado las relaciones cambiantes entre un PANE (ICV) y su partido hermano a nivel estatal (IU) a lo largo de las últimas décadas. Las dimensiones propuestas para el análisis del grado de integración vertical entre los distintos niveles territoriales de los partidos bajo sistemas de gobierno multinivel resultan también útiles para estudiar el marco de cooperación entre PAE y PANE. Dada la autonomía total de ICV en cuanto a las cuestiones organizativas, la formulación de la oferta programática y el diseño de las estrategias electorales y la política de alianzas, la articulación de las relaciones las asemejaba en el período 1987-1997 a un partido bifurcado, con lo cual disminuía un paso más en la integración vertical que mantenían los partidos predecesores, el PCE y el PSUC, cuya posición en el continuum estaba más cerca de los partidos confederales.

La soberanía absoluta de ICV respecto a IU en estas dimensiones fue una fuente constante de conflictos desde la creación de ambos partidos a mediados de los años ochenta y el factor determinante en la ruptura total de relaciones. A partir de ese momento, ICV se constituyó en un partido truncado, puesto que se quedó sin un referente en el ámbito estatal. Poco después, cada organización iniciaba un nuevo recorrido. ICV estrechaba los lazos con los partidos verdes a través de una federación estatal e IU impulsaba la creación de una nueva contraparte en Cataluña, EUiA.

Los pésimos resultados electorales obtenidos desde 1999, en los que la ruptura fue sin duda un factor importante, condujeron a una nueva aproximación que se tradujo, a partir de 2002, en una coalición entre ICV y EUiA que persiste hasta la fecha. No se ha reestablecido ningún marco de cooperación

39. IPV terminó escindiéndose de EUPV por desacuerdo con las listas electorales aprobadas para las elecciones generales de 2008. La dirección de IU, a pesar de mantener una mayor sintonía con IPV, se vio obligada a apoyar a EUPV para evitar mayores fracturas en el conjunto de la organización. 
en las esferas organizativas, ideológicas o políticas de alianzas entre ICV e IU (a través de su referente en Cataluña) más allá de los imperativos que dicta la coalición electoral.

A su vez, a través del estudio de la cooperación interpartidista multinivel, podemos plantear algunas hipótesis sobre los factores que afectan al grado y a las formas de cooperación entre la unidad estatal y las unidades subestatales que deberán ser comprobadas en el análisis de otros casos (UPN-PP, PSCPSOE). En primer lugar, una integración vertical alta es viable exclusivamente cuando existe una elevada coincidencia en las políticas desarrolladas por ambos. En momentos de divergencia, aparecen tendencias que buscan una mayor autonomía y la consiguiente reacción en el PAE, que busca homogeneizar las posiciones. En segundo lugar, la fortaleza electoral del PANE también es una variable muy relevante. Cuanto mayor sea ésta, mayor autonomía exigirá el PANE respecto al PAE. En tercer lugar, unas relaciones personales tensas entre los responsables de ambos partidos, en especial si el líder del PANE tiene un carácter marcadamente autonomista, erosionan el grado de cooperación.

El examen de las relaciones entre IU e ICV nos ha permitido analizar también la evolución ideológica y organizativa de ICV desde su creación. Los distintos caminos ideológicos y estratégicos emprendidos por las dos organizaciones trajeron resultados dispares y paradójicos. En primer lugar, un éxito electoral del PANE y una erosión electoral constante del PAE y, en segundo lugar, tras la aproximación producida desde el año 2000 a raíz del cambio de liderazgo en IU, un papel influyente de ICV sobre IU. Como hemos visto, en la legislatura 2004-2008, ICV representaba el 40\% del grupo parlamentario federal en el Congreso de los Diputados. La actual dirección de IU ha tomado a ICV como una experiencia exitosa a emular para refundar su proyecto político, a fin de proyectar una nueva imagen pública de compromiso con la izquierda verde y de fuerza responsable de gobierno.

No obstante, entre mediados de 2008 y principios de 2009, se celebrarán congresos en todos los partidos (ICV, EUiA, PCE e IU), lo que puede tener un impacto significativo en estas relaciones en función de los nuevos liderazgos, especialmente tras la nueva debacle electoral de IU. En las elecciones generales de marzo de 2008 , IU vio reducida su representación a dos diputados, e ICV, a uno, por lo que fue necesario alcanzar un pacto con ERC para contar con grupo parlamentario propio. Habrá que ver, pues, cómo afrontan las dos organizaciones la igualdad en la representación institucional y cómo se ven afectadas las relaciones en esta nueva etapa en la que, además, aunque Gaspar Llamazares sigue en el Congreso de los Diputados, ya no dirige la coordinación general de IU, que ha pasado a manos del PCE. 


\section{Referencias bibliográficas}

Astudillo, Javier; GARCía GUERETA, Elena (2005). «La distribución territorial del poder en los partidos políticos: El caso del Partido Popular español», ponencia presentada en el VII Congreso de la AECPA. Madrid, 21-24 de septiembre.

Botella, Joan (2003). «From Red to Green: The Evolution of Catalan Communism». En: Joan Botella y Luis RAMIRO-FernáNDEZ (eds.). The Crisis of Communism and Party Change. The Evolution of West European Communist and Post-communist Parties. Barcelona: ICPS, p. 53-67.

CEBRIÁN, Carme (1997). Estimat PSUC. Barcelona: Empúries.

ChHibBer, Pradeep; Kollman, Ken (2004). The Formation of National Party Systems: Federalism and Party Competition in Canada, Great Britain, India and the United States. Oxford: Princeton University Press.

DesCHOWER, Kris (2003). «Political Parties in Multi-layered Systems». European Urban and Regional Studies, n. ${ }^{\circ}$ 10, vol. 3, p. 213-26.

Downs, William M. (1998). Coalition Government Subnational Style. Columbus: Ohio State University Press.

DunPHY, Richard (2004). Contesting Capitalism? Left Parties and European Integration. Manchester: Manchester University Press.

EstruCH, Joan (1982). El PCE en la clandestinidad: 1939-1956. Madrid: Siglo XXI.

GOMÀ, Ricard; RIUS, Marc (2006). «Iniciativa per Catalunya Verds. La izquierda verde de Catalunya». En: Ángel Valencia SÁrZ (ed.). La izquierda verde. Barcelona: Nous Horitzons-Icària.

HeLLER, William B. (2002). «Regional parties and national politics in Europe». Comparative Political Studies, n. ${ }^{\circ}$ 35, vol. 6, p. 657-85.

HopKIN, Jonathan; BRADBURY, Jonathan (2006). «British Statewide Parties and MultiLevel Politics». Publius: The Journal of Federalism, n. ${ }^{\circ} 36$, p. 135-52.

JandA, Kenneth (1980). Political Parties: A Cross-National Survey. Nueva York: The Free Press.

MÉNDEZ-LaGo, Mónica; OrTe, Andreu (2005). «La organización de partidos en sistemas multinivel: el caso del PSOE», ponencia presentada en el VII Congreso de la AECPA. Madrid: Universidad Complutense, 21-24 de septiembre.

Pallarès, Francesc; KeAting, Michael (2003). «Multi-level Electoral Competition: Regional Elections and Party Systems in Spain». European Urban and Regional Studies, n. ${ }^{\circ} 10$, vol. 3, p. 239-55.

Panebianco, Angelo (1988). Political Parties: Organization and Power. Cambridge: Cambridge University Press.

Paniagua, Juan L.; RAMIRO-Fernández, Luis (2003). Voz, conflicto y salida. Un estudio sobre faccionalismo: Nueva Izquierda, 1992-2001. Madrid: Editorial Complutense.

RAmiro-FernÁNDEZ, Luis (2004). «Electoral Competition, Organizational Constraints and Party Change: The Communist Party of Spain (PCE) and United Left (IU), 1986-2000». Journal of Communist and Transition Politics, n. ${ }^{\circ} 20$, vol. 2, p. 1-29.

- (2005). "The Crisis of the Spanish Radical Left: The PCE and IU. A Rejoinder». Journal of Communist and Transition Politics, n. ${ }^{\circ} 21$, vol. 2, p. 303-5.

RAMIRO-FERNÁNDEZ, Luis; PérEZ-NieVAS, Santiago (2005). «El impacto de los procesos de descentralización territorial en la organización de los partidos políticos: el caso de Izquierda Unida», ponencia presentada en el VII Congreso de la AECPA. Madrid: Universidad Complutense, 21-24 de septiembre. 
RENZSCH, Wolfgang (2001). «Bifurcated and Integrated Parties in Parliamentary Federations: The Canadian and German Cases». Working Paper 2001/4. Queens University.

RiBÓ, Rafael (1999). Una altra Catalunya, una altra esquerra. Barcelona: Pòrtic.

RIUS, Marc (2005). «Iniciativa per Catalunya Verds (1987-2004). Una història de l'esquerra verda de Catalunya». Quaderns Pensament i l'Acció, n. ${ }^{\circ} 15$, Nous Horitzons.

Roller, Elisa; VAn Houten, Pieter (2003). "National Party in a Regional Party System: The PSC-PSOE in Catalonia». Regional and Federal Studies, n. ${ }^{\circ}$ 13, vol. 3, p. 1-21.

SAURA, Joan (2000). «Rol de l'esquerra verda nacional». Quaderns Pensament i l'Acció, n. ${ }^{\circ}$ 3, Nous Horitzons.

Smiley, Donald (1987). The Federal Condition in Canada. Toronto: Mc-Graw-Hill Ryerson.

Thorburn, Hugh G. (1991). Party Politics in Canada. Scarborough: Prentice Hall.

THORLAKSON, Lori (2001). «Federalism and party organisational adaptation: a crossnational comparison", ponencia presentada en el ECPR Joint Sessions of Workshops. Grenoble, Francia, 6-11 de abril.

ThORLAKSON, Lori (2006). «Party Systems in Multi-level Contexts». En: Dan HougH y Charles JefFERY (eds.). Devolution and Electoral Politics. Manchester: Manchester University Press.

VÁZquez Montalbán, Manuel (1988). Rafael Ribó: l'optimisme de la raó. Barcelona: Planeta.

Vega, Pedro; Erroteta, Peru (1982). Los herejes del PCE. Barcelona: Planeta.

VERGE, Tània (2007). «Is United Left becoming a party of government?», ponencia presentada en la Political Science Association. Bath, Reino Unido, 11-13 de abril.

\section{Listado de acrónimos}

CiU: Convergència i Unió.

ERC: Esquerra Republicana de Catalunya.

EUiA: Esquerra Unida i Alternativa.

ICV: Iniciativa per Catalunya Verds.

IU: Izquierda Unida.

PAE: Partido de ámbito estatal.

PANE: Partido de ámbito no estatal.

PCC: Partit dels Comunistes de Catalunya.

PCE: Partido Comunista de España.

PP: Partido Popular.

PSC: Partit dels Socialistes de Catalunya.

PSOE: Partido Socialista Obrero Español.

PSUC: Partit Socialista Unificat de Catalunya.

PSUC viu: Partit Socialista Unificat de Catalunya viu.

UPN: Unión del Pueblo Navarro. 